\title{
Protometabolic Reduction of $\mathrm{NAD}^{+}$with $\alpha$-Keto Acids
}

Shibaji Basak, Serge Nader, and Sheref S. Mansy*

Cite This: JACS Au 2021, 1, 371-374

Read Online

ACCESS | Llll Metrics \& More | 回 Article Recommendations | (s Supporting Information

ABSTRACT: Deciphering the origins of the chemistry that supports life has frequently centered on determining prebiotically plausible paths that produce the molecules found in biology. What has been less investigated is how the energy released from the breakdown of foodstuff is coupled to the persistence of the protocell. To gain better insight into how such coupled chemistry could have emerged prebiotically, we probed the reactivity of the ribodinucleotide $\mathrm{NAD}^{+}$with small organic molecules that were previously identified as potential constituents of protometabolism.

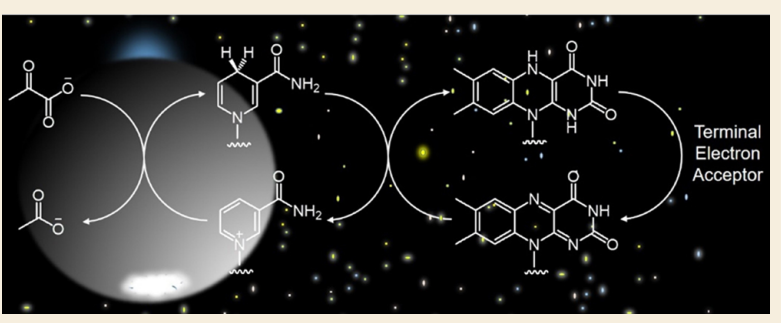
We find that $\mathrm{NAD}^{+}$is readily reduced nonenzymatically by $\alpha$-keto

acids, such as pyruvate and oxaloacetate, during oxidative decarboxylation. In the presence of FAD and a terminal electron acceptor, the consumption of $\alpha$-keto acids by $\mathrm{NAD}^{+}$initiates a plausible prebiotic electron transport chain. The observed reactivity suggests that components of the RNA world were capable of initiating the chemistry needed to capture the energy released from catabolism to drive anabolism.

KEYWORDS: protometabolism, protocell, $\mathrm{NAD}^{+}$, oxidative decarboxylation, RNA world

7 he primary role of metabolic chemistry is to maintain the L low entropy state of the cell. The discovery of nonenzymatic reactions similar to those found in metabolism based on the identity of substrates and products is useful in that such work indicates what types of chemistry were potentially accessible on the prebiotic Earth. ${ }^{1}$ However, such prebiotic chemistry would also have been inconsequential if not coupled to the thermodynamically unfavorable reactions needed to sustain the cell. Extant biology does this, in large part, by plugging into oxidative chemistry through the electron shuttling activity of nicotinamide adenine dinucleotide. To date, no examples of prebiotic, metabolic-like chemistry have demonstrated the capturing of electrons with prebiotically plausible electron shuttling molecules.

Despite the considerable focus placed on the RNA world, ${ }^{2-4}$ little attention has been given to the redox active dinucleotides found in biology. Fragments of the prebiotic synthesis of $\mathrm{NAD}^{+}(\mathbf{1})$ were reported, ${ }^{5-7}$ and 1 can engage in electron transfer with iron-sulfur peptides, ${ }^{8}$ rhodium trisbipyridine complexes, ${ }^{9,10}$ and titanium dioxide particles. ${ }^{11}$ Further, a laboratory evolved ribozyme can couple the oxidation of a benzyl alcohol to the reduction of $\mathbf{1}$ to $\mathrm{NADH}(2) .{ }^{12}$

We sought to determine whether $\mathrm{NAD}^{+}(1)$ could have engaged in catabolic-like reactions that could have ultimately driven cell-like activity (Scheme 1). Here, we show that $\mathbf{1}$ can acquire electrons from $\alpha$-keto acids, including pyruvate, during oxidative decarboxylation. Several prebiotic routes for the synthesis of pyruvate have been reported. ${ }^{13-15}$ Subsequently, the produced NADH (2) nonenzymatically reduces FAD to $\mathrm{FADH}_{2}$. Finally, the electrons are deposited into a model
Scheme 1. Electron Transfer from $\alpha$-Keto Acids

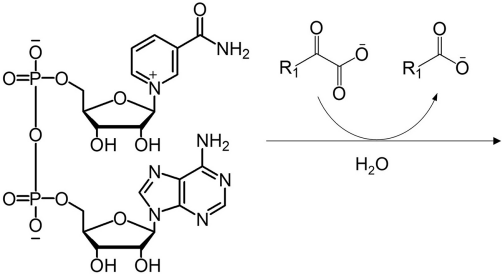

1

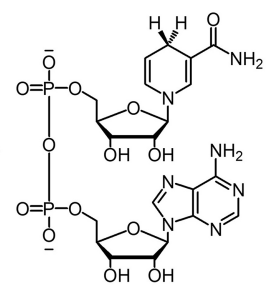

2 terminal electron acceptor, methylene blue. Similar electron transport chains may have impacted the prebiotic chemistry that became metabolism.

When $\mathrm{NAD}^{+}$(1) was incubated with pyruvate (3) at $23{ }^{\circ} \mathrm{C}$ at $\mathrm{pH} 9.0$ for $24 \mathrm{~h},{ }^{1} \mathrm{H}$ NMR spectra showed a decrease in the resonances of $\mathbf{1}$ and an increase in resonances that corresponded to acetate (4) and NADH (2) (Figures 1a and S1). Integration of the peaks revealed that the starting concentrations of $1(15 \mathrm{mM})$ and $3(30 \mathrm{mM})$ decreased to 8.1 and $19.7 \mathrm{mM}$, respectively. After $24 \mathrm{~h}, 0.9 \mathrm{mM} 4$ and 1.0

Published: March 12, 2021

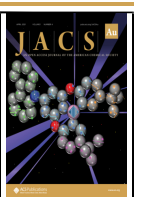


a)

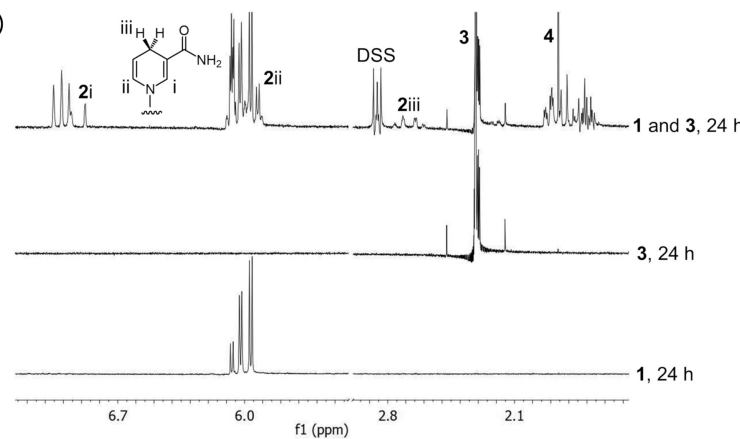

b)
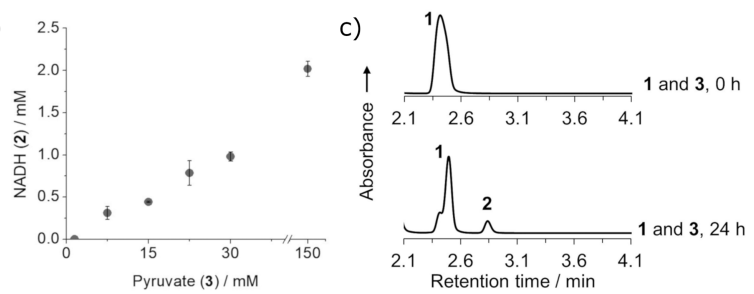

Figure 1. (a) ${ }^{1} \mathrm{H}$ NMR spectra of the reaction products from incubation of 1 and 3 showed formation of 2 and 4. (b) The reduction of $1(15 \mathrm{mM})$ to 2 with $3(24 \mathrm{~h}) .3$ was at 1.5, 7.5, 15, 22.5, 30 , and $150 \mathrm{mM}$. Data are mean and SEM (standard error of the mean) of distinct samples, $n=2$. (c) HPLC chromatograms show the formation of 2 after $24 \mathrm{~h}$. All reactions were in $200 \mathrm{mM}$ bicarbonate, $\mathrm{pH} 9.0$.

mM 2 formed, indicating that $<10 \%$ of the consumed 3 and 1 led to the formation of 4 and 2. Pyruvate (3) was not consumed under the same experimental conditions in the absence of 1 (Figure S2). Despite the inefficiency of the reaction, reduction of $\mathbf{1}$ to $\mathbf{2}$ strongly correlated with the production of $4(\rho=0.95)$ (Figure S3), consistent with a dependence on the oxidative decarboxylation of 3 . The presence of 4 was confirmed by $1 \mathrm{D}$ and $2 \mathrm{D}$ heteronuclear NMR with ${ }^{13} \mathrm{C}$ labeled 3 (Figures S4-S6). The production of 2 was confirmed by UV-vis absorption and fluorescence spectrophotometry (Figure S7). After $1 \mathrm{~h}$, an absorption band at $340 \mathrm{~nm}$ and an emission band at $450 \mathrm{~nm}$ emerged that were consistent with the presence of 2 . After $24 \mathrm{~h}$, an additional absorption band appeared at $430 \mathrm{~nm}$, and the emission peak shifted to $505 \mathrm{~nm}$, suggesting the formation of degradation products derived from 1. LC-MS of these samples (Figure S8) corroborated the production of $2(\mathrm{~m} / z=664.1168$, $\left.\mathrm{C}_{21} \mathrm{H}_{29} \mathrm{~N}_{7} \mathrm{O}_{14} \mathrm{P}_{2}-\mathrm{H}^{-}\right)$after 1 and $24 \mathrm{~h}$. Since NADH (2) can exist in two different major isomeric forms (1,4-NADH and $1,6-\mathrm{NADH})$ that can be difficult to distinguish, the identity of 2 was verified by the enzymatic activity of lactate dehydrogenase, ${ }^{10}$ which only mediates the oxidation of $1,4-\mathrm{NADH}$. After incubation of $\mathbf{1}$ and 3 for $24 \mathrm{~h}$, the addition of lactate dehydrogenase led to an immediate loss of the resonances of the produced 2 (Figure S9). Taken together, the data were consistent with the nonenzymatic reduction of 1 to 2 by 3 .

The formation of NADH (2) was dependent on the concentration of pyruvate (3) (Figures $1 \mathrm{~b}$ and S10) with a Pearson correlation coefficient of 0.94 . A $150 \mathrm{mM}$ concentration of 3 reduced $13.4 \pm 0.6 \%$ of $15 \mathrm{mM} \mathrm{NAD}^{+}$(1) to $\mathrm{NADH}$ (2) after $24 \mathrm{~h}$. Lower concentrations of 3 reduced less 1 under the same experimental conditions (Figure 1b). For example, $30 \mathrm{mM} 3$ led to the reduction of $6.5 \pm 0.4 \%$ of 1 to 2 . Similarly, titrations of $\mathbf{1}$ with a constant concentration of 3 showed a direct correlation between 1 and $3(\rho=0.99)$, as expected (Figure S11).

Reduction of $\mathrm{NAD}^{+}$(1) to NADH (2) by pyruvate (3) was dependent on $\mathrm{pH}$ (Figure S12). 2 was not produced below $\mathrm{pH}$ 8. Since the reactions at $\mathrm{pH} 7.5$ and 8.0 were in $200 \mathrm{mM}$ sodium phosphate and the reactions at higher $\mathrm{pH}$ were in 200 $\mathrm{mM}$ bicarbonate, it was not clear if the increased reactivity at higher $\mathrm{pH}$ was due to $\mathrm{pH}$ or participation of the buffer. Therefore, 1 was incubated with 3 in $200 \mathrm{mM}$ pyrophosphate, $\mathrm{pH} 9.0$, which led to the reduction of $5.2 \pm 0.9 \%$ of 1 to 2 (Figure S14). The data were within error of the $6.5 \pm 0.4 \%$ observed in $200 \mathrm{mM}$ bicarbonate. Thus, the buffer did not play a significant role. At $\mathrm{pH}$ above 7.0, some degradation of 1 was observed (Figure S15a), with $26.0 \pm 3.5 \%$ of $\mathbf{1}$ degraded at $\mathrm{pH}$ 9 after $24 \mathrm{~h}$. Conversely, no degradation of 3 was detected by ${ }^{1} \mathrm{H}$ NMR spectroscopy over the same $\mathrm{pH}$ range. Further, no differences in the equilibrium between 3 , hydrated-3, and the formation of parapyruvate were detected (Figure S15b). Reactions at higher temperatures were not pursued because of the increased degradation of 1 (Figure S16). Ambient light did not facilitate the reduction of $\mathbf{1}$ (Figure S16). ${ }^{16}$

Since $\alpha$-keto acids undergo facile decarboxylation, ${ }^{17-19}$ the ability of $\alpha$-keto acids other than pyruvate (3) to reduce $\mathrm{NAD}^{+}$ (1) was investigated. The incubation of 1 with oxaloacetate (5), $\alpha$-ketoglutarate (6), 2-ketobutyrate (7), and 2-oxooctanoate (8) all gave rise to NADH (2) (Figures 2 and S17). However, the yield of $\mathbf{2}$ was greater with $\mathbf{3}$ and 7 . For example, $74 \%$ less 2 was produced at $\mathrm{pH} 9.0$ with 5 than with 3 . All

a)

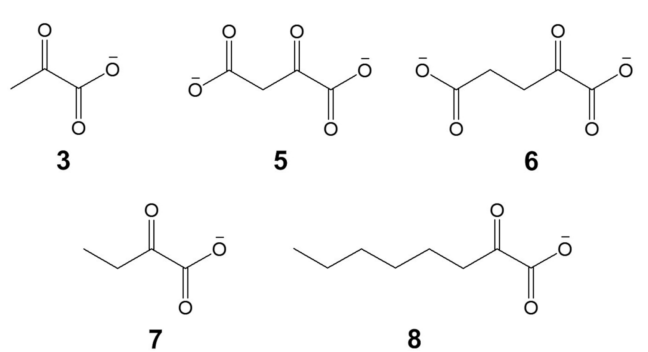

b)

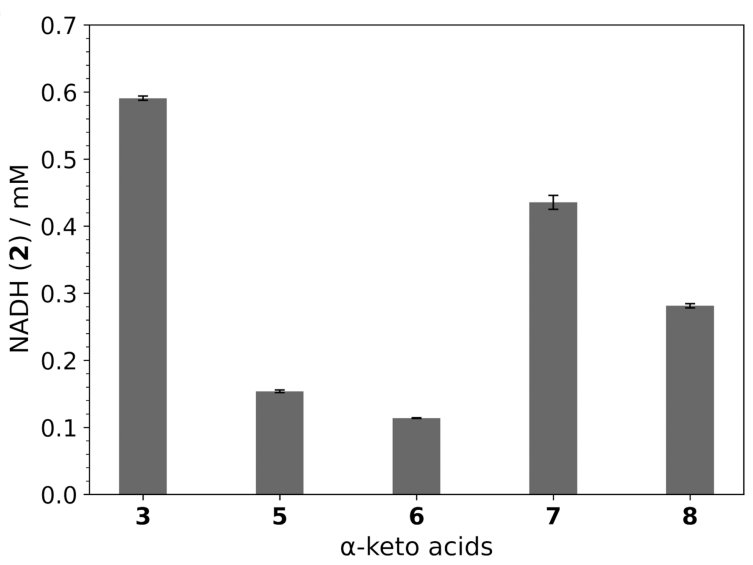

Figure 2. (a) Chemical structures of tested $\alpha$-keto acids. (b) Reduction of 1 to 2 by $\alpha$-keto acids. A $30 \mathrm{mM}$ concentration of each $\alpha$-keto acid (3 and 5-8) was incubated with $1(15 \mathrm{mM})$ for $24 \mathrm{~h}$ at $\mathrm{pH}$ 9.0. Analysis was by HPLC. Data are mean and SEM of distinct samples, $n=2$. 
reactions with $\alpha$-keto acids showed increased activity at higher $\mathrm{pH}$ (Figure S12). The ${ }^{1} \mathrm{H}$ NMR spectra of reactions with 5 were similar to reactions with 3 , because 5 decarboxylates to 3 (Figure S18). ${ }^{20}$ Decreased yields of $\mathbf{2}$ with $\mathbf{5}$ and $\mathbf{6}$ suggest that the additional carboxylate interferes with the mechanism, perhaps by raising the $\mathrm{p} K_{\mathrm{a}}$ of the enol. The decreased reactivity of 8 likely stemmed from the formation of aggregates. ${ }^{21}$

Next, we investigated the importance of the $\alpha$-carbonyl by testing the activity of molecules with a $\beta$-carbonyl (acetoacetic acid, 9) and a $\gamma$-carbonyl (levulinic acid, 10) (Figures S19 and S20). Both molecules failed to reduce detectable amounts of $\mathrm{NAD}^{+}(\mathbf{1})$ as judged by ${ }^{1} \mathrm{H}$ NMR spectroscopy. The activity of glyoxylate (11), which contains an $\alpha$-aldehyde as opposed to an $\alpha$-ketone, was also evaluated. Glyoxylate (11) was unable to reduce 1 to 2 (Figure S21). Finally, other components of the citric acid cycle that enzymatically provide electrons to 1 , such as malate (12) and isocitrate (13), were tested. Neither 12 nor 13 were capable of reducing 1 to 2 (Figure S22). Of the molecules tested, only enolizable $\alpha$-keto acids were found to nonenzymatically reduce 1 to 2 , possibly through a single electron transfer mechanism (Figures S23 and 24), although other mechanisms are possible.

In addition to $\mathrm{NAD}^{+}(\mathbf{1}), \mathrm{FAD}(14)$ is a redox active ribodinucleotide that may have played a role in early protocellular systems (Figure S25). ${ }^{22}$ In extant biology, $\mathrm{NADH}$ (2) frequently reduces flavins, e.g., $\mathrm{NADH}$ dehydrogenase. Consistent with previous reports of the nonenzymatic reduction of 14 by 2,12 the electrons from pyruvate $(3)$ were shuttled to 14 via $\mathrm{NAD}(\mathrm{H})$ (Figure 3a). More specifically, if 3

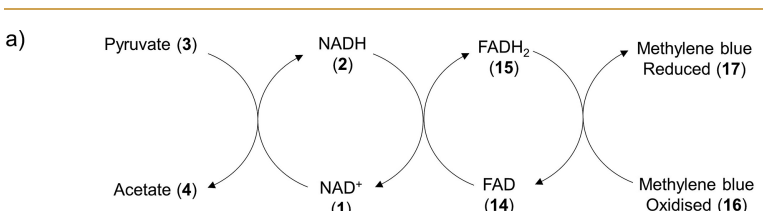

b)

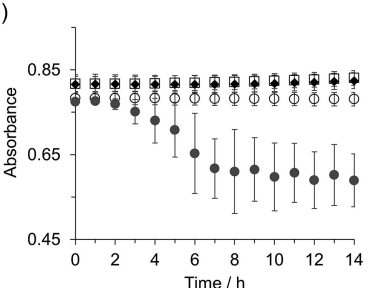

c)

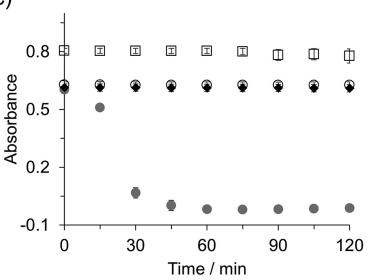

Figure 3. (a) Assembly of an electron transport chain. (b) The reduction of $14(0)$. The reaction contained $30 \mathrm{mM} \mathrm{3,15} \mathrm{mM} \mathrm{1,} 0.1$ $\mathrm{mM} \mathrm{14}$, and $200 \mathrm{mM}$ bicarbonate, $\mathrm{pH} 9.0$, and was monitored at 475 $\mathrm{nm}$. The reaction did not proceed in the absence of $1(O)$ or $3(\checkmark)$ or in the presence of the nonactive electron donor 9 ( $\square$ ). (c) The transfer of electrons from 3 to $16(\bullet)$. Reactions contained $30 \mathrm{mM} 3$, $15 \mathrm{mM} \mathrm{1,7.5} \mathrm{mM} \mathrm{14,} 13.3 \mu \mathrm{M} \mathrm{16}$, and $200 \mathrm{mM}$ bicarbonate, $\mathrm{pH} 9.0$. 16 was not reduced in the absence of either $1(\checkmark), 3(\bigcirc)$, or $14(\square)$. Data are mean and SEM of distinct samples, $n=2$.

was premixed and incubated with $\mathbf{1}$ for $24 \mathrm{~h}$, the ${ }^{1} \mathrm{H}$ resonances of $\mathbf{2}$ diminished to undetectable levels upon the addition of $\mathbf{1 4}$ (Figure S26). If all three, i.e., 1, 3, and 14, were incubated together from the beginning, ${ }^{1} \mathrm{H}$ NMR spectra showed a clear dependence on the amount of $\mathrm{FADH}_{2}(\mathbf{1 5})$ produced on the starting concentration of $\mathbf{1}$ (Figure S27). UV-vis absorption spectroscopy corroborated the reduction of $\mathbf{1 4}$ to $\mathbf{1 5}$ (Figures $3 \mathrm{a}$ and S28). 14 absorbs strongly, whereas 15 is colorless. ${ }^{23}$
The reaction was complete within $8 \mathrm{~h}$, and no reduction of $\mathbf{1 4}$ was observed in the absence of $\mathbf{3}$ or $\mathbf{1}$. Similarly, $\mathbf{1 4}$ was not reduced if $\mathbf{3}$ was replaced with a nonactive electron donor, e.g., the $\beta$-keto acid 9 (Figure 3a).

The electron transport chain was completed with a model terminal electron acceptor, methylene blue (16). Consistent with the electron shuttling roles of $\mathrm{NAD}^{+}(\mathbf{1})$ and $\mathrm{FAD}(14)$, the electrons from pyruvate (3) ultimately reduced 16 (Figures $3 \mathrm{~b}$ and S29) after incubation for $120 \mathrm{~min} .16$ was not reduced when either $3, \mathbf{1}$, or $\mathbf{1 4}$ were not included in the reaction mixture. A more prebiotically plausible terminal electron acceptor would be ferricyanide. ${ }^{24}$ However, the use of ferricyanide would require spatial separation, since ferricyanide can oxidize 2 and 15. Ferricyanide did not oxidize 3 under the exploited conditions (Figure S30).

In the modern-day citric acid cycle, one $\alpha$-keto acid $(\alpha$ ketoglutarate, (6)) and two $\alpha$-hydroxy acids (malate (12) and isocitrate $(13))$ are oxidized in a manner that reduces $\mathrm{NAD}^{+}$ (1) to NADH (2). ${ }^{25,26}$ Of these reactions, only 6 was found to reduce $\mathrm{NAD}^{+}$(1) nonenzymatically, albeit inefficiently. However, oxaloacetate (5) was able to reduce 1 nonenzymatically, even if $\mathbf{5}$ is not oxidatively decarboxylated, and $\mathbf{5}$ does not reduce $\mathbf{1}$ in the contemporary citric acid cycle. Therefore, if a prebiotic, $\mathrm{NAD}^{+}$-dependent metabolic-like pathway existed, this pathway would likely have been different from what is observed in biology today. One possibility is that prebiotic analogues of contemporary metabolism more fully dependent on $\alpha$-keto acids. ${ }^{27,28}$ For example, a nonenzymatic $\alpha$-keto acid version of the citric acid cycle was recently reported. ${ }^{28}$ Although this cycle runs in reverse, using glyoxylate to reduce $\alpha$-keto acids, each of the $\alpha$-keto acid intermediates would be capable of reducing 1 . Therefore, even if glyoxylate does not reduce $\mathrm{NAD}^{+}$directly, the electrons from glyoxylate could be transferred through $\alpha$-keto acids to $\mathrm{NAD}^{+}$, thus initiating a thermodynamically favorable electron transport chain. Another possibility is that $\mathrm{NAD}^{+}$could be reduced by $\mathbf{5}$ through the activity of a prebiotic, $\alpha$-keto acid-dependent glyoxylate cycle. ${ }^{27}$ This cycle was reported to proceed via the oxidative decarboxylation of $\mathbf{5}$ with hydrogen peroxide. $\mathbf{1}$ can mediate the same reaction without the loss of electrons. Further efforts in combining the redox active components of the RNA world with oxidative, metabolic-like chemistry will likely give critical insights into the neglected but necessary nonenzymatic steps between abiotic and biotic chemistry.

\section{ASSOCIATED CONTENT}

Supporting Information

The Supporting Information is available free of charge at https://pubs.acs.org/doi/10.1021/jacsau.0c00124.

NMR, UV-vis absorption, fluorescence, LC-MS, and HPLC data of all the compounds and controls (PDF)

\section{AUTHOR INFORMATION}

\section{Corresponding Author}

Sheref S. Mansy - Department of Chemistry, University of Alberta, Edmonton, AB T6G 2G2, Canada; $\odot$ orcid.org/ 0000-0003-2382-198X; Email: sheref.mansy@ualberta.ca

\section{Authors}

Shibaji Basak - Department of Chemistry, University of Alberta, Edmonton, AB T6G 2G2, Canada; $\odot$ orcid.org/ 0000-0002-8790-0209 
Serge Nader - Department of Chemistry, University of Alberta, Edmonton, AB T6G 2G2, Canada; (1) orcid.org/ 0000-0002-1472-3918

Complete contact information is available at: https://pubs.acs.org/10.1021/jacsau.0c00124

\section{Author Contributions}

S.B. and S.N. performed the experiments. S.S.M. supervised the work. The manuscript was written through contributions of all authors.

Funding

We acknowledge the support of the Simons Foundation (290358FY19 to S.S.M.) and the Natural Sciences and Engineering Research Council of Canada (NSERC) [RGPIN-2020-04375 to S.S.M.].

Notes

The authors declare no competing financial interest.

\section{ACKNOWLEDGMENTS}

We thank J. S. Sutherland for helpful suggestions throughout the course of the work, R. Krishnamurthy for insightful comments on the manuscript, and S. Alladin-Mustan for assistance with HPLC.

\section{ABBREVIATIONS}

$\mathrm{NAD}$, nicotinamide adenine dinucleotide; $\mathrm{FAD}$, flavin adenine dinucleotide

\section{REFERENCES}

(1) Ruiz-Mirazo, K.; Briones, C.; de la Escosura, A. Prebiotic systems chemistry: new perspectives for the origins of life. Chem. Rev. 2014, 114, 285-366.

(2) Joyce, G. F. The antiquity of RNA-based evolution. Nature 2002, 418, 214-221.

(3) Szostak, J. W.; Bartel, D. P.; Luisi, P. Luigi Synthesizing life. Nature 2001, 409, 387-390.

(4) Gilbert, W. Origin of life: The RNA world. Nature 1986, 319, 618.

(5) Kim, H.-J.; Benner, S. A. A Direct Prebiotic Synthesis of Nicotinamide Nucleotide. Chem. - Eur. J. 2018, 24, 581-584.

(6) Huang, F.; Bugg, C. W.; Yarus, M. RNA-Catalyzed CoA, NAD, and $\mathrm{FAD}$ synthesis from phosphopantetheine, NMN, and FMN. Biochemistry 2000, 39, 15548-15555.

(7) Cleaves, H. J.; Miller, S. L. The Nicotinamide Biosynthetic Pathway Is a By-Product of the RNA World. J. Mol. Evol. 2001, 52, 73-77.

(8) Bonfio, C.; Godino, E.; Corsini, M.; Fabrizi de Biani, F.; Guella, G.; Mansy, S. S. Prebiotic iron-sulfur peptide catalysts generate a $\mathrm{pH}$ gradient across model membranes of late protocells. Nat. Catal. 2018, $1,616-623$.

(9) Abril, O.; Whitesides, G. M. Hybrid Organometallic/Enzymatic Catalyst Systems: Regeneration of NADH Using Dihydrogen. J. Am. Chem. Soc. 1982, 104, 1552-1554.

(10) Dalai, P.; Sahai, N. A Model Protometabolic Pathway across Protocell Membranes Assisted by Photocatalytic Minerals. J. Phys. Chem. C 2020, 124, 1469-1477.

(11) Summers, D. P.; Rodoni, D. Vesicle Encapsulation of a Nonbiological Photochemical System Capable of Reducing $\mathrm{NAD}^{+}$to NADH. Langmuir 2015, 31, 10633-10637.

(12) Tsukiji, S.; Pattnaik, S. B.; Suga, H. An alcohol dehydrogenase ribozyme. Nat. Struct. Mol. Biol. 2003, 10, 713-717.

(13) Cody, G. D.; Boctor, N. Z.; Filley, T. R.; Hazen, R. M.; Scott, J. H.; Sharma, A.; Yoder, H. S., Jr. Primordial carbonylated iron-sulfur compounds and the synthesis of pyruvate. Science 2000, 289, 13371340.

(14) Cooper, G.; Reed, C.; Nguyen, D.; Carter, M.; Wang, Y. Detection and formation scenario of citric acid, pyruvic acid, and other possible metabolism precursors in carbonaceous meteorites. Proc. Natl. Acad. Sci. U. S. A. 2011, 108, 14015-14020.

(15) Coggins, A.; Powner, M. Prebiotic synthesis of phosphoenol pyruvate by $\alpha$-phosphorylation-controlled triose glycolysis. Nat. Chem. 2017, 9, 310-317.

(16) Rapf, R. J.; Dooley, M. R.; Kappes, K.; Perkins, R. J.; Vaida, V. $\mathrm{pH}$ Dependence of the Aqueous Photochemistry of $\alpha$-Keto Acids. J. Phys. Chem. A 2017, 121, 8368-8379.

(17) Hanson, R. W. J. Decarboxylation of $\alpha$-Keto Acids. J. Chem. Educ. 1987, 64, 591-595.

(18) Nwaukwa, S. O.; Keehn, P. M. Oxidative cleavage of $\alpha$-diols, $\alpha$ diones, $\alpha$-hydroxy-ketones and $\alpha$-hydroxy- and $\alpha$-keto acids with calcium hypochlorite $\left[\mathrm{Ca}(\mathrm{OCl})_{2}\right]$. Tetrahedron Lett. 1982, 23, 31353138.

(19) Moon, P. J.; Lundgren, R. J. Metal-Catalyzed Ionic Decarboxylative Cross-Coupling Reactions of $\mathrm{C}\left(\mathrm{sp}^{3}\right)$ Acids: Reaction Development, Mechanisms, and Application. ACS Catal. 2020, 10, $1742-1753$.

(20) Jencks, W. P. Catalysis in Chemistry and Enzymology; McGrawHill: New York, 1969; vol. 116.

(21) Xu, H.; Du, N.; Song, Y.; Song, S.; Hou, W. Vesicles of 2ketooctanoic acid in water. Soft Matter 2017, 13, 2246-2252.

(22) Rivas, M.; Becerra, A.; Lazcano, A. On the Early Evolution of Catabolic Pathways: A Comparative Genomics Approach. I. The Cases of Glucose, Ribose, and the Nucleobases Catabolic Routes. J. Mol. Evol. 2018, 86, 27-46.

(23) Oprian, D. D.; Coon, M. J. Oxidation-reduction states of FMN and FAD in NADPH-cytochrome P-450 reductase during reduction by NADPH. J. Biol. Chem. 1982, 257, 8935-8944.

(24) Xu, J.; Ritson, D. J.; Ranjan, S.; Todd, Z. R.; Sasselov, D. D.; Sutherland, J. D. Photochemical reductive homologation of hydrogen cyanide using sulfite and ferrocyanide. Chem. Commun. 2018, 54, $5566-5569$.

(25) Donaldson, R. P.; Fang, T. K. $\beta$-oxidation and glyoxylate cycle coupled to NADH: cytochrome $\mathrm{c}$ and ferricyanide reductases in glyoxysomes. Plant Physiol. 1987, 85, 792-795.

(26) Duncan, R. J. S.; Tipton, K. F. The oxidation and reduction of glyoxylate by lactic dehydrogenase. Eur. J. Biochem. 1969, 11, 58-61.

(27) Springsteen, G.; Yerabolu, J. R.; Nelson, J.; Rhea, C. J.; Krishnamurthy, R. Linked cycles of oxidative decarboxylation of glyoxylate as protometabolic analogs of the citric acid cycle. Nat. Commun. 2018, 9, 91.

(28) Stubbs, R. T.; Yadav, M.; Krishnamurthy, R.; Springsteen, G. A plausible metal-free ancestral analogue of the Krebs cycle composed entirely of $\alpha$-ketoacids. Nat. Chem. 2020, 12, 1016-1022. 